Received: November 2, 2017

Revision received: March 7, 2018

\title{
Research on Mathematical Education and Teaching Design Based on Brain Science*
}

\author{
Junhui Mo ${ }^{1}$ \\ Zhengsong $\mathrm{Yu}^{2}$ \\ Linyuan $\mathrm{Wei}^{3}$ \\ Anyang Normal University \\ Anyang Normal University \\ Henan University
}

\begin{abstract}
The research findings of brain science have been widely used in various fields. The in-depth study of brain science can help to develop brain potential and creativity efficiently, thus effectively improving the quality of teaching. In order to optimize mathematics teaching, this paper conducts the research on brain science-based mathematics education and teaching design. Through the theoretical analysis of brain science and the analysis for complex relationships in the process of mathematics teaching, combined with the characteristics of mathematics, this paper discusses the relationship between symbolic mathematics, situational mathematics and linguistic mathematics and its application in mathematics education, and also explores the mathematics teaching design and implementation based on brain science. The research results of this paper shall provide scientific theoretical support for creating a suitable mathematics teaching environment, and further promote the scientific and efficient development of mathematics teaching.
\end{abstract}

\section{Keywords}

Brain Science • Mathematics Education • Teaching Design

\footnotetext{
* The work was funded by National Natural Science Foundation of China (Grant No: 41601177); The Ministry of Education of Humanities and Social Science Project (Grant No: 15YJCZH212); The Project of Young Backbone Teachers in Henan Province (Grant No:2017GGJS127)

${ }^{1}$ School of Mathematics and Statistics, Anyang Normal University, Anyang 455000, China. Email: mojunhui@sohu.com.

${ }^{2}$ Correspondence to: School of Environment Resources and Tourism Management, Anyang Normal University, Anyang 455000, China. Email: yuzhengsong220@163.com

${ }^{3}$ School of Education, Henan University, Kaifeng 475001, China. Email: 17839164205@163.com
}

Citation: Mo, J. H., Yu, Z. S. \& Wei, L. Y, Mother-Child and Teacher-Child Relationships and Their Associations with School Adjustment in Pre-school. Educational Sciences: Theory \& Practice, 18(5), 1432-1439. http://dx.doi.org/10.12738/estp.2018.5.040 
As of the "Decade Brain" project in the 1990s, brain science research has made great progress, and the development prospects of brain science and its significance to human beings have attracted the attention of the scientific community and governments. The research results of brain science have been widely used in various fields, and in-depth research in brain science can help to develop brain potential and creativity efficiently. The results of brain science research play an important role in the field of education. On the one hand, it is used to evaluate the scientific nature of various quality education practices; on the other hand, it can also be used to provide excellent teaching methods for quality education (Marope 2016).

The brain is an important material carrier for people's cognitive learning. The development of brain science promotes the in-depth study of mathematics learning and teaching, and there exists a close relationship between brain science and mathematics teaching. In general, for complex mathematics education and learning problems, the related educator and learners of mathematics need to clearly discover certain mathematical laws and further achieve better teaching and learning. Although there are interactions between mathematics learning and brain cognition, in some brain science research results, the brain learning mechanism has systematically and deeply studied, but they have not been effectively applied in mathematics education learning (Poo, Xu and Tan, 2016). As an emerging field, brain science is still based on basic research, and parents and educators do not know much about brain science. In addition, brain science research specifically designed to solve mathematics education and classroom learning needs further study. Limited research results limit the popularization and application of brain science in the field of education. Therefore, the research results and theories of brain science play an important role in improving the quality of modern mathematics education.

The brain can carry all kinds of human knowledge and reflect the objective world while building the subjective world. Mathematics is the reflection of the human brain based on subjective and objective things, and then builds operational tools that form knowledge, intelligence, and abilities. There are always two different voices in the mathematics education community: the scholars with the background in psychology and education recommend the use of living and situational mathematics, while those with mathematics professional background recommend mathematizing and symbolic mathematics. However, with the continuous innovation and improvement of brain science cognition and theory, the study on brain mechanism of mathematical cognition provides powerful brain science evidence for the integration of contextualized and symbolic mathematics (Hall, 2005). In this context, for optimizing mathematics teaching, this paper conducts the research on brain science-based mathematics education and teaching design. Through the theoretical analysis of brain science and the analysis for complex relationships in the process of mathematics teaching, combined with the characteristics of mathematics, this paper discusses the relationship between symbols, situations, and language and its application in mathematics education. In addition, it also discusses the design and implementation of mathematics classroom teaching based on the results of brain science research. The research results of this paper provide scientific theoretical support for creating a suitable mathematics teaching environment, which plays an important role and guiding significance for improving the quality of mathematics education.

This paper consists of five chapters. Chapter 1 is the introduction. Chapter 2 discusses the related content of brain science and mathematics teaching. Chapter 3 carries out the application research of brain science in 
Mo, Yu, Wei / Mother-Child and Teacher-Child Relationships and Their Associations with School Adjustment in Pre-school

mathematics education. The fourth chapter makes mathematics teaching design based on brain science. The last chapter is the conclusion.

\section{Brain science and mathematics teaching}

\section{Theoretical analysis of brain science}

The theory of brain science describes the working mechanism of the common activities by neuronal cells in the brain, analyses the causes of environmental stimuli and effects, and further theoretically describes the advanced cognitive behaviours of the brain, mainly including language, intelligence, thinking and perception behaviour, etc. (Gu and Shi 2015). The brain is the most complex structural organization in the human body, and to reveal how the brain works has become the biggest challenge in modern brain science. The cerebral cortex is the centre of the brain's advanced functions, and is divided into four areas, such as the parietal lobe, occipital lobe, temporal lobe and frontal lobe. The functions of these areas are clearly different, e.g., the temporal lobe responsible for auditory and memory functions, the occipital lobe responsible for visual function, the frontal lobe responsible for intellectual function, and the parietal lobe for somatosensory integration function. Despite the different divisions of labour in these regions, studies have shown that many advanced functions require the participation of different regions (Foster, Lyte, Meyer and Cryan, 2016). At present, the main research focus of brain science have turned to the cellular and molecular mechanisms of advanced brain function, and the study of learning and memory is quite profound. The results show that the cell level of longterm memory and short-term memory is accompanied by the improvement of neuronal excitability and changes in neurotransmitter release. In addition, brain science theory has also achieved some research results in the development of brain intellectual potential (Fjell, Westlye and Amlien, 2015), whole brain development and brain function localization (Yang and Chang, 2014), brain functional structure etc. Thinking mode and cognition are regarded as the core of brain science education. Thus, cognitive innovation and learning training based on brain science has become a new direction for educational practitioners.

\section{Analysis of complex relationships in the process of mathematics teaching}

The intrinsic link between the brain and mathematics is the most relevant discipline and knowledge in all subject teaching and knowledge systems. Therefore, for better mathematics teaching, it is necessary to deal with the complex relationship in the process of mathematics teaching, and then realize the integration of mathematics teaching and brain science through full use of brain science. This section analyses the complex relationship in the process of mathematics teaching from three aspects: intelligence factor, thinking factor and teaching relationship.

Intelligence is a comprehensive cognitive ability, which mainly includes basic factors such as attention, memory, thinking, observation and imagination. Abstract thinking ability and creativity can be regarded as the core and highest performance of intelligence. In the process of complex mathematics learning activities, various intellectual factors and non-intellectual factors affect the learning process. The involvement of intellectual factors in the process of psychological cognition directly affects the learning activities of mathematics. Non- 
Mo, Yu, Wei / Mother-Child and Teacher-Child Relationships and Their Associations with School Adjustment in Pre-school

intellectual factors do not directly participate in the process of psychological cognition, but indirectly affect the efficiency of mathematics learning activities. A number of studies have shown that students' academic performance and intelligence have certain correlation. Intelligence factors have an impact on academic achievement and mathematics learning, and also affect the flexibility, speed and depth of students' knowledge learning. The difference between the innate quality and the acquired conditions of each person makes the difference in intelligence. On the one hand, intelligence differences are reflected in differences of intelligence levels and that in early and late performance; on the other hand, intelligence differences are reflected in differences of intelligence types and gender. Therefore, intellectual differences have a great influence on students' academic achievement and mathematics learning to some extent.

Abstract thinking ability is a kind of ability for people to understand the essence of things, and it is also an important ability to form revealing laws and scientific understanding. Abstraction is the process of thinking that extracts the common features of things. Abstractness is the basic feature of mathematics. The teaching and learning of mathematics require a strong abstract thinking. Because of the different brain functional regions, mathematics culture is a culture of truth-seeking, different from the discipline of language arts. Mathematical teaching should reflect the abstraction of thinking and the generalization of knowledge. Also, it needs to be expressed by mathematical symbols and solve specific problems through mathematical modelling. In the process of mathematics teaching and learning, teachers should combine the discipline characteristics of mathematics and its teaching value to cultivate students' abstract thinking ability. If students pay too much attention to the image and picture, this will not be conducive to the formation of students' abstract thinking ability and affect the growth speed of the brain. In the process of mathematics teaching and learning, the mathematics cognition and wisdom shall be further developed by teaching mathematics knowledge, culture, thinking and methods to develop students' intelligence.

Teaching is the process of teaching and learning. In the teaching process, teachers and students cooperate in order to accomplish common teaching goals. This process reflects the harmonious unity of teacher teaching and student learning, including the teacher-student interaction, common development, and active interaction. Teaching is usually composed of four factors: teachers, students, teaching methods, and teaching content which interact and influence each other to form a unity of opposites. As said in the Chinese saying, it is better to teach people to fish than to teach people to fish. The teacher's responsibility is to teach students the tools of fishing, let the students go fishing, rely on themselves to discover, and increase the knowledge. Due to the rapid development of mathematics science, the goal of mathematics teaching should be to enable students to learn self-study mathematics. Therefore, teaching is aimed at learning. The teaching of teachers and the learning of students are the main contradiction in the teaching process, and they play a decisive role in restricting other contradictions in the teaching process.

\section{Applied research of brain science in mathematics education}

There are two different viewpoints of mathematics education method in mathematics education community. One method focuses on learning mathematics in specific situations, emphasizes the contextualization of mathematics, and the other focuses on constructing a rigorous knowledge system of mathematics, highlighting 
Mo, Yu, Wei / Mother-Child and Teacher-Child Relationships and Their Associations with School Adjustment in Pre-school

the symbolization of mathematics. Some scholars have realized that the two must be integrated with each other and also proposed corresponding methods (Dong, Zhang and Zhou, 2005). The fusion of contextualization and symbolization requires an in-depth study of mathematical cognition in brain science. Mathematical contextualization is closely related to speech comprehension and contextual memory, more focusing on solving mathematical problems in specific life contexts, while the mathematical symbolization is more focused on symbol processing, quantity processing and mathematical calculation reasoning. The brain mechanism of mathematical context is related to the temporal lobe, frontal lobe, hippocampus and other regions, and the brain mechanism of mathematical symbolization is mainly located in the parietal lobe region. In mathematics cognition, some brain regions have close functional connections, so mathematical activities can be seen as the result of joint action by multiple brain regions. Mathematical symbols and mathematical situations are consistent with the laws of brain activity.

In the process of mathematics education and learning, the contextualized content such as digital symbolic calculation, reasoning and other symbolic content processing and mathematical application questions will activate the regions of brain speech processing. Speech processing plays an important role in the understanding of mathematical problems and principles, so both symbolic and situational mathematics require semantic network and speech processing. On the one hand, the brain activation patterns in semantic coding are almost the same, so the situation and language have certain correlation, and some components are connected. In the process of mathematical understanding, the specific language content can be extracted from the problem situation memory, and the problem situation memory can also be activated by the semantic network. On the other hand, the processing of mathematical terms relies mainly on the semantic brain regions in language elements, and the solution to mathematical problems such as application questions etc. also depends on the brain semantic network. Therefore, it is necessary to combine the unique roles and interrelationships of language mathematics, situational mathematics and symbolic mathematics in mathematics education and learning, and then carry out ternary mathematics education practice based on brain science theory.

As shown in Fig.1, ternary mathematics education considers the fusion of symbolic mathematics, situational mathematics, and linguistic mathematics. Symbolic mathematics refers to the use of mathematical symbols such as numbers and letters to represent mathematical principles; situational mathematics refers to situations that contain mathematical principles; linguistic mathematics refers to the description of mathematical principles in natural language. These three have complementary relationships that transform each other and promote mathematical understanding and memory. Specifically, the mathematical knowledge system framework through language description was used to solve the incomplete problem of mathematical knowledge system construction. At the same time, the language advantages were adopted to understand the abstract mathematical symbols, and then establish the mathematical symbol system supported by mathematical situation so as to solve the problem of difficult understanding caused by focusing on symbolization.

Mathematical principles are the core of mathematics teaching and learning, and also the basis of mathematical situations and mathematical symbols. Symbolic mathematics, situational mathematics, and linguistic mathematics should be cantered on understanding mathematical knowledge and applying mathematical principles. Mathematical formulas, rules, theorems and other mathematical principles are mainly used for the logical judgment of the relationship between concept attributes and concepts. The mathematical 
symbols and language are related to the parietal region and the frontal lobe region respectively, and the arithmetic principle processing depends on these regions, indicating that the mathematical principle learning requires the joint action of the three.

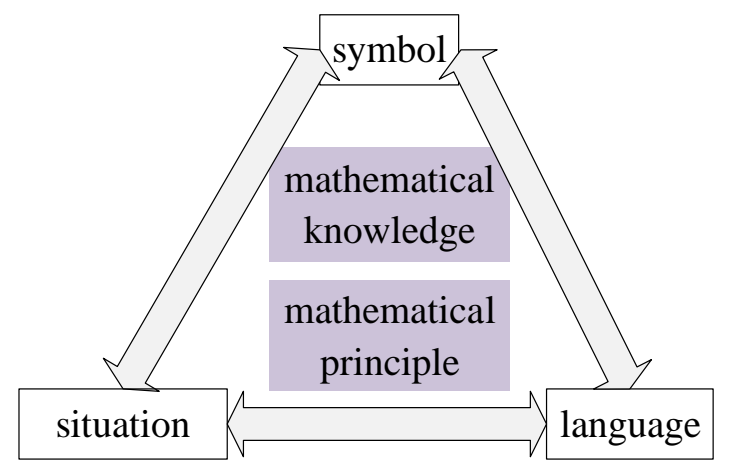

Figure 1. Ternary mathematics education

In the teaching process of ternary mathematics education, it can start with the situational mathematics teaching, or with the symbolic mathematics teaching. The former describes the mathematical theories involved in the specific situation in words and expresses the mathematical problems in mathematical symbols. The latter uses specific speech to describe the meaning and grammar of mathematical symbols, and then introduces mathematical problems into specific mathematical situations. Both ideas are the result of the transformation among the three elements of symbol, situation and language, and the process of their mutual transformation is reversible. For the same mathematical problem, this means that words can be used to describe the specific situation of the problem, and words that describe mathematical problems and principles can be transformed into mathematical symbols. Since the functions for mathematical symbol processing and speech description are in the frontal lobe and temporal lobe of the brain, and the functions of these regions are bidirectionally connected, speech description, situation mathematics, and symbolic mathematics are integrated and complementary to each other in the process of mathematics teaching. In addition, the main goal of mathematics education should be the mutual conversion between linguistic mathematics, situational mathematics, and symbolic mathematics. Therefore, the corresponding mathematics teaching evaluation criteria should also consider the three dimensions of speech, situation and symbol, and must include the ability of solving problems in specific situations and mathematical understanding.

\section{Mathematical teaching design based on brain science}

In the current mathematics education process, students have heavy learning burden and low efficiency, and there also exist the widespread phenomena such as insufficient ability to acquire mathematics knowledge and lack of mathematical application ability. Therefore, it is very important to strengthen the integration of research results in brain science with the teaching process of mathematics. In the process of mathematics teaching design, more emphasis should be put on three aspects: visual space ability training, brain memory system training and student estimation ability training. 
Mo, Yu, Wei / Mother-Child and Teacher-Child Relationships and Their Associations with School Adjustment in Pre-school

First of all, mathematics teaching focuses on students' visual space ability training. The research results of brain science show that the extraction of arithmetic knowledge and the formulation of algorithm rule play an important role in solving complex mathematical calculation problems, and the students need language representation and visual spatial representation for storage learning (Chen, Wu and Cheng, 2006). Therefore, in the actual mathematics classroom teaching process, teachers need to use more rigorous language logic to adopt the method of knowledge teaching. The study found that the visual spatial function is related to the upper part of the left parietal lobe, e.g., during the calculation process, the abacus masters produced more activation in the region, indicating that the use of more visual spatial functions helps to improve students' computing power.

Second, mathematics teaching should insist on brain memory system training. The results of brain science research show that the activation of brain regions involved in mathematical calculations will change with the increase of individual age and training (Rotzer, Kucian and Martin, 2008). The parietal regions of students with strong mathematical computing skills are fully developed, so these students have more grey matter in the region of the right intraparietal sulcus. These findings provide important insights into the development of students' mathematical abilities. Therefore, the ability to process and store digital information is especially important for mathematics education. In the mathematics classroom teaching process, the student's working memory breadth can be trained by numbers, to activate the ability of extracting arithmetic information in long-term memory.

Finally, mathematics teaching should strengthen students' estimation ability. The research results of brain science show that actuarial tasks rely on specific linguistic representations, while estimation tasks rely on the visual spatial representation of numbers (Qi and Zhang 2002). The brain region activated by the actuarial task significantly overlaps with the language area, and the estimation task is closely related to the relevant areas of physical perception and spatial perception. Therefore, both estimation ability and actuarial ability play an important role in the development of individual computing power. In the actual mathematics classroom teaching process, if only the actuarial ability is emphasized, the lack of estimation ability will result in the mathematical thinking without flexibility and even mathematical common sense. Unlike the training of actuarial abilities, estimation ability training should be linked to spatial, visual, and motor perception training.

\section{Conclusion}

The research results of brain science play an important role in the field of education, and the theoretical research of brain science helps to develop brain potential and creativity efficiently. This paper focuses on mathematics education and teaching design research based on brain science. Firstly, it analyses the complex relationship of intelligence, thinking and teaching in the process of brain science and mathematics teaching. Then, combined with the characteristics of mathematics, it discusses the relationship between symbols, situations and language in mathematics education, and also its specific application. Finally, from the three aspects of visual space ability training, brain memory system training and student estimation ability training, it explores the mathematics teaching design based on brain science. The mathematics education and teaching design research results of this paper can be used well in the construction of mathematics teaching environment, providing practical teaching methods and scientific theoretical support. 


\section{References}

Marope, M. (2016). "Brain science, education, and learning: Making connections," Prospects, 46, 87-190.

Poo, M., Xu, B., Tan, T. (2016). "Brain Science and Brain-Inspired Intelligence Technology-An Overview," Bulletin of Chinese Academy of Sciences, 31(7), 725-736, http://dx.doi.org/10.16418/j.issn.10003045.2016.07.001

Hall, J. (2005). A review of the contribution of brain science to teaching and learning.

Gu, S., Shi, F. (2015). "Controllability of structural brain networks," Nature communications, 6, http://dx.doi.org/10.1038/ncomms9414

Foster, J., Lyte, M., E. Meyer, J. (2016). Cryan, "Gut Microbiota and Brain Function: An Evolving Field in Neuroscience," International Journal of Neuropsychopharmacology, 19(5), 1-114, http://dx.doi.org/10.1093/ijnp/pyv114

Fjell, A., Westlye, L., Amlien, I. (2015). "High-expanding cortical regions in human development and evolution are related to higher intellectual abilities," Cerebral Cortex, 25(1), 26-34, http://dx.doi.org/10.1093/cercor/bht201

Yang, Z., Chang, C. (2014). "Connectivity trajectory across lifespan differentiates the precuneus from the default network,” NeuroImage, 89(1), 46-56, http://dx.doi.org/10.1016/j.ncuroimagc.2013.10.039

Dong, Q., Zhang, H., Zhou, X. (2005). "Mathematical cognition: research findings and educational implications of brain and cognitive science," Journal of Beijing Normal University, 3, 40-46.

Chen, C., Wu, T., Cheng, M. (2006). "Prospective demonstration of brain plasticity after intensive abacus-based mental calculation training: An fMRI study," Nuclear Inst \& Methods in Physics Research A, 569(2), 567571, http://dx.doi.org/10.1016/j.nima.2006.08.101

Rotzer, S., Kucian, K., Martin, E. (2008). "Optimized voxel-based morphometry in children with developmental dyscalculia," $\quad$ Neuroimage, $39(1), \quad 417-422$, http://dx.doi.org/10.1016/j.neuroimage.2007.08.045

Qi, D. Q., Zhang, H. (2002). "Estimation ability and actuarial ability: research results of brain and cognitive science and their implications for mathematics education," Educational Research, 5, 46-51. 